\title{
Global SH-wavefield calculation for a two-dimensional whole- Earth model with the parallel hybrid PSM/FDM algorithm
}

\author{
Yanbin Wang • Yang Luo • Yanfang Qin • \\ Xianbing Zhang
}

Received: 9 December 2013/Accepted: 11 April 2014/Published online: 15 May 2014

(c) The Seismological Society of China, Institute of Geophysics, China Earthquake Administration and Springer-Verlag Berlin Heidelberg 2014

\begin{abstract}
We present a parallel hybrid algorithm based on pseudospectral method (PSM) and finite difference method (FDM) for two-dimensional (2-D) global SHwavefield simulation. The whole-Earth model is taken as a cross section of spherical Earth, and corresponding wave equations are defined in 2-D cylindrical coordinates. Spatial derivatives in the wave equations are approximated with efficient and high accuracy PSM in the lateral and high-order FDM in the radial direction on staggered grids. This algorithm allows us to divide the whole-Earth into sub-domains in radial direction and implement efficient parallel computing on PC cluster, while retains high accuracy and efficiency of PSM in lateral direction. A transformation of moment tensor between 3-D spherical Earth and our 2-D model was proposed to give corresponding moment tensor components used in 2-D modeling. Comparison of modeling results with those obtained by direct solution method shows very good accuracy of our algorithm. We also demonstrate its feasibility with a lateral heterogeneous whole-Earth model with localized velocity perturbation.
\end{abstract}

Y. Wang $(\bowtie) \cdot$ X. Zhang

Department of Geophysics, School of Earth and Space Sciences, Peking University, Beijing 100871, China

e-mail: ybwang@pku.edu.cn

\section{Y. Luo}

Department of Geosciences, Princeton University,

312 Guyot Hall, Princeton, NJ 08544, USA

Y. Qin

Equipe de Géosciences Marines, Institut de Physique du Globe de Paris, 4 Place Jussieu, 75252 Paris Cedex 05, France
Keywords SH-wave propagation · Whole-Earth · Numerical modeling · Hybrid method · Parallel computing

\section{Introduction}

Seismological investigation of the Earth's interior has been improved with growing high quality broadband seismic observations. Seismic waveform analysis by comparing observations with synthetics is one of the most effective approaches for study of the Earth's inner structure. For teleseismic applications, synthetic waveforms for a large range of epicentral distances are generally calculated for a lateral heterogeneous whole-Earth model with numerical method. For examples, Cummins et al. (1997) applied the direct solution method (DSM) to compute complete synthetic seismograms for a laterally heterogeneous spherical whole-Earth model and illustrated effects of two-dimensional (2-D) (axisymmetric) upper-mantle heterogeneity on SH-wavefield. Takeuchi et al. (2000) modified the DSM operators and calculated synthetic waveforms for spherical whole-Earth model with three-dimensional (3-D) heterogeneity and showed their applications in waveform inversion. With steady increase of computational performance, full 3-D global seismic waveform modeling became available on parallel hardware. Komatitsch and Tromp (2002a, 2002b) developed a spectral-element method to simulate seismic wave propagation in the whole spherical Earth with full complexity of 3-D heterogeneity.

The full 3-D modeling of large scale global seismic wave propagation is still computationally intensive due to large computer memory and a long computation time and is not efficient for routine-like study of the Earth's structure. Therefore, numerical methods have been developed for 2-D realistically simplified whole-Earth model to save 
computational resources and improve efficiency. By assuming that structure heterogeneity is invariant in the out of plane direction, an axisymmetric 2-D whole-Earth model is often used in global seismology as a good approximation of the 3-D spherical Earth. Nissen-Meyer et al. (2007a, 2007b) developed a 2-D spectral-element method for computing the full 3-D moment-tensor and point force response of a spherically symmetric earth model in a 2-D semi-circular computational domain. Several studies have applied finite difference method (FDM) to such a 2-D global model. Igel and Weber $(1995,1996)$ used a high-order FDM to a 2-D axisymmetric whole-Earth model to calculate $\mathrm{SH}$ and $\mathrm{P}-\mathrm{SV}$ wave propagations, respectively, with lateral heterogeneity. Igel and Gudmundsson (1997) applied this method and studied frequencydependent effects of long-period S and SS waves. Thorne et al. (2007) performed 2-D global SH-wavefield simulation based on FDM to study lateral variation in the lowermantle structure beneath the Cocos Plate. Jahnke et al. (2008) presented a 2-D axisymmetric global SH wave modeling for use on a parallel computer and examined the comparability of a ring source to a double-couple source. Toyokuni et al. (2005) and Toyokuni and Takenaka (2006, 2012) proposed a new FDM approach for 2-D axisymmetric model in the "quasi-spherical domain" and the implementation of moment-tensor point source and anelastic attenuation. Wen (2002) developed a hybrid method for SH wavefield simulation including 2-D localized heterogeneous structures by combining generalized ray theory and FDM and studied shear velocity structures in the lowermost mantle beneath the central Pacific and South Atlantic Oceans.

Comparing with FDM, the Fourier pseudospectral method (PSM) provides an alternative spatial derivative operator that can achieve the same accuracy as FDM but with less grid points and hence requires less computer memory and computation time (Fornberg 1996). Furumura et al. (1998) applied PSM to simulate global P-SV wave propagation for the 2-D whole-Earth model defined in cylindrical coordinates. Wang et al. $(2001,2010)$ extended this method to a whole 2-D Earth model including the center by solving numerical problems associated with the singularity at the center of the Earth. Wang and Takenaka (2011) presented a PSM scheme to simulate SH-wave propagation in a 2-D whole-Earth model with arbitrary lateral heterogeneities. Wang et al. (2013) presented numerical modeling of seismic wave propagation for P-SV wavefield in a whole Moon model with a hybrid method based on PSM and FDM.

In this paper, we present a hybrid numerical algorithm combining PSM and FDM for global SH-wavefiled simulation. The 2-D whole-Earth model is defined in a cylindrical coordinate and field variables in wave equations are discretized with staggered grids. Spatial derivatives in wave equations are calculated with PSM operator in the lateral direction, and with FDM in the radial direction. Such a hybrid PSM/FDM algorithm enables us to divide the whole-Earth model into sub-domains along radial direction and perform efficient parallel modeling on PC clusters. In order to consider moment-tensor source in the 2-D model, we propose a rotation scheme of moment tensor between spherical and cylindrical coordinates to get the corresponding components of moment tensor for 2-D model. We will first introduce the hybrid PSM/FDM algorithm, its parallel implementation on PC cluster and the rotation of moment tensor. Then, we test the accuracy of this algorithm by comparing synthetic global seismograms calculated by our method with DSM results for Preliminary Reference Earth Model (PREM) (Dziewonski and Anderson 1981). Finally, we demonstrate feasibility of our algorithm by applying it to a whole-Earth model with lateral shear velocity perturbation just above the coremantle boundary (CMB).

\section{Wave equations and hybrid PSM/FDM algorithm}

\subsection{Wave equations}

We consider SH wave propagation in a whole-Earth model. In a cylindrical coordinate system $(r, \theta, z)$, assuming invariance in $z$ for all fields, the equation of momentum conservation for transverse $\mathrm{SH}$-waves in velocity-stress form is given by:

$\rho \frac{\partial v_{z}}{\partial t}=\frac{\partial \sigma_{z r}}{\partial r}+\frac{1}{r}\left(\frac{\partial \sigma_{z \theta}}{\partial \theta}+\sigma_{z r}\right)+f_{z}$,

The constitutive relations between stress and velocity for isotropic and elastic medium are:

$$
\begin{gathered}
\frac{\partial \sigma_{z r}}{\partial t}=\mu \frac{\partial v_{z}}{\partial r}, \\
\frac{\partial \sigma_{z \theta}}{\partial t}=\frac{\mu}{r} \frac{\partial v_{z}}{\partial \theta},
\end{gathered}
$$

where $v_{z}$ is the transverse velocity, $f_{z}$ is the body force, $\sigma_{z r}$ and $\sigma_{z \theta}$ are stress tensor components. $\rho$ and $\mu$ represent mass density and shear modulus of the medium.

\subsection{Hybrid PSM/FDM algorithm}

We consider a 2-D cross-section cutting through a great circle of the spherical Earth, which is defined between the Earth's surface and outer core in the radial $(r)$ and $0^{\circ}-360^{\circ}$ in the lateral $(\theta)$ direction. In order to solve the above equations for such a model with hybrid PSM/FDM algorithm, we first discretize the computational domain with 


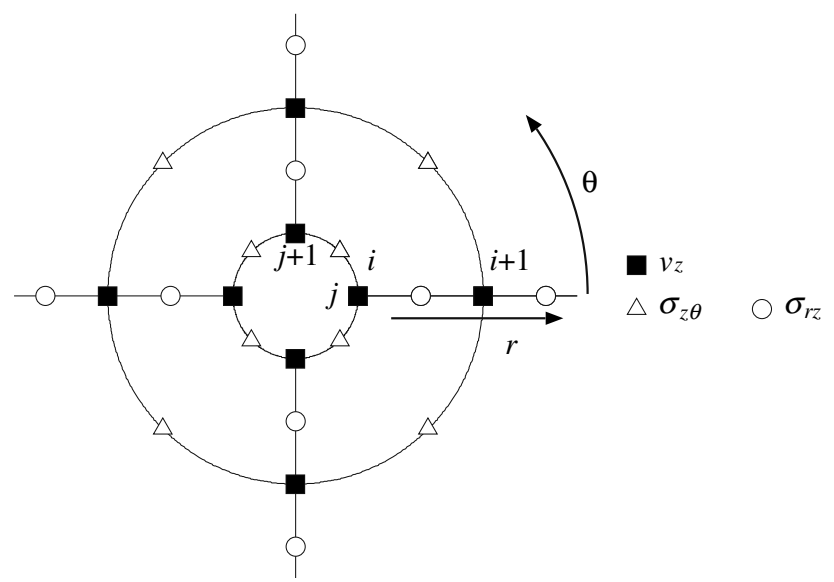

Fig. 1 Layout of discretized grid points for the staggered grid algorithm for global SH wavefield modeling in 2-D cylindrical coordinates $(r, \theta) . i$ and $j$ are grid points in $r$ and $\theta$ directions

staggered grids along both lateral and radial directions as schematically illustrated in Fig. 1. The velocity, stress components, and medium parameters are defined at different locations with half grid spacing between each other.

Wang and Takenaka (2011) presented a PSM scheme to solve global $\mathrm{SH}$ wave propagation for this model. They discretized the whole model with non-staggered grid and approximated spatial derivatives in both $r$ and $\theta$ directions with PSM operator. However, this scheme could potentially bring numerical artifacts arising from sharp discontinuity of medium parameter due to the global nature of PSM. Hence, they discretized the model with irregular grid spacing in the $r$ direction and treated free surface boundary with air-filled zone in order to suppress numerical artifacts. This scheme is difficult to be implemented on a parallel hardware due to the global derivative operator and limits the computational efficiency.

In order to suppress potential numerical artifacts and improve computational efficiency, we here propose the hybrid PSM/FDM algorithm to solve above wave equations. In $\theta$ direction, we use PSM operator for staggered grids to calculate spatial derivatives. This will naturally incorporate the periodic boundary condition in $\theta$ direction for the whole-Earth model in the calculation. This operation was proved to be efficient in reducing numerical artifacts arising from sharp discontinuity as compared with non-staggered grid PSM (Witte and Richards 1990; Özdenva and McMechan 1996). Let $M$ represents the grid number in $\theta$ direction, the corresponding discrete field variable is $f(j \Delta \theta), j=0,1,2, \ldots, M-1$ (Fig. 1). It is first transformed into wavenumber domain as $F(l \Delta k), l=0,1$, $2, \ldots, M-1$ via a $1-\mathrm{D}$ fast Fourier transformation (FFT):

$F(l \Delta k)=\frac{1}{M} \sum_{j=0}^{M-1} f(j \Delta \theta) \mathrm{e}^{-i 2 \pi j l / M}$
The spatial derivative is then calculated half-way between grid points $((j \pm 1 / 2) \Delta \theta)$ in the wavenumber domain by multiplication and inverse transformation via 1D FFT (Wang and Takenaka 2010):

$$
\begin{aligned}
& \frac{\mathrm{d}}{\mathrm{d} \theta} f\left[\left(j \pm \frac{1}{2}\right) \Delta \theta\right]=\frac{\Delta k}{2 \pi} \sum_{l=0}^{M-1} i(l \Delta k) \mathrm{e}^{ \pm i l \Delta k \Delta \theta / 2} F(l \Delta k) \mathrm{e}^{i 2 \pi j l / M} \\
& j=0,1,2, \ldots, M-1
\end{aligned}
$$

In $r$ direction, we use a fourth-order staggered grid FDM to approximate spatial derivatives. Let $f(i \Delta r), i=0,1,2$, ..., $N-1$ represents a discrete field variable in $r$ direction with $N$ grid points (Fig. 1), the spatial derivatives on internal grids can be calculated by (Virieux 1986):

$$
\begin{aligned}
\frac{\mathrm{d}}{\mathrm{d} r} f(i \Delta r)= & \frac{1}{\Delta r}\left\{c_{1}\left[f\left(\left(i+\frac{1}{2}\right) \Delta r\right)-f\left(\left(i-\frac{1}{2}\right) \Delta r\right)\right]\right. \\
& \left.+c_{2}\left[f\left(\left(i+\frac{3}{2}\right) \Delta r\right)-f\left(\left(i-\frac{3}{2}\right) \Delta r\right)\right]\right\}, \\
& i=0,1,2, \ldots, N-1
\end{aligned}
$$

where $c_{1}=9 / 8, c_{2}=1 / 24$. In the staggered grid configuration shown in Fig. 1, $\sigma_{z r}$ at the right hand side of Eq. (1) is calculated with a Fourier interpolation method (Wang and Takenaka 2001).

Since the localized FDM derivative operator is used in $r$ direction, the free surface boundary condition at the Earth's surface is treated by satisfying traction-free condition and additional air zone is not necessary. At the bottom of the model in the outer core, we apply absorbing boundaries with a 20-grid buffer zone (Cerjan et al. 1985) to reduce artificial reflections. The velocity field $v_{z}$ in wave equations is calculated in time domain with a second order FDM approximation (Furumura et al. 1998). The time interval $\Delta t$ is determined from the stability condition defined by the minimum grid spacing $\Delta_{\min }$ and the maximum shear velocity $V_{\max }$ in the whole model:

$\Delta t \leq \alpha \frac{\Delta_{\min }}{V_{\max }}$

where parameter $\alpha$ is 0.606 for FDM (Daudt et al. 1989) and 0.45 for PSM (Fornberg 1996), respectively. We take a value of 0.45 to ensure stability for both PSM and FDM in the hybrid algorithm.

\subsection{Parallel Implementation on PC Cluster}

The stagger-grid FDM used in $r$ direction is a localized operation. It enables us to divide the 2-D whole-Earth model into sub-domains along radial direction. All subdomains have the same number of grid points in both $\theta$ and $r$ directions. We assign each sub-domain to a single processor of a PC cluster to perform parallel computing. Since 
a fourth-order FDM on staggered grid is used, we add two layers of grids above and below each sub-domain named as overlap regions. The neighboring processors exchange quantities in the overlap domain for calculating derivatives with respect to the radial coordinate $(r)$. These values sent/ received between sub-domains then used in FDM to update current wavefields. The inter-processor communication and data exchange is carried out by the message passing interface (MPI). Since a localized FDM operator is used, work space required for data exchange between subdomains is negligible. Qin et al. (2012) and Furumura et al. (2002) applied a PSM/FDM algorithm to solve seismic wave equations defined in 3-D Cartesian coordinates and simulated regional seismic wave propagation from the 1999 Chi-Chi (Ji-Ji) earthquake. They found that the hybrid PSM/FDM offers a good speed-up rate using a large number of processors.

The way in which we solve wave Eqs. (1) and (2) with the parallel hybrid PSM/FDM algorithm is accomplished by the following procedures:

(1) Divide the 2-D whole-Earth model into sub-domains along $r$ direction and assign them to processors of PC cluster.

(2) In each processor, calculate spatial derivatives of velocity field with respect to $\theta\left(\partial v_{z} / \partial \theta\right)$ with PSM and to $r\left(\partial v_{z} / \partial r\right)$ with FDM. Data exchange between subdomains for overlapping regions.

(3) Calculate stress components $\left(\sigma_{z r}, \sigma_{z \theta}\right)$ in Eq. (2) with time domain differencing.

(4) In each processor, calculate spatial derivatives of stress field with respect to $\theta\left(\partial \sigma_{z \theta} / \partial \theta\right)$ with PSM and to $r\left(\partial \sigma_{z r} / \partial r\right)$ with FDM. Data exchange between subdomains for overlapping regions.

(5) Calculate velocity fields $\left(v_{z}\right)$ in Eq. (1) with time domain differencing.

(6) The next time step.

\subsection{Incorporation of moment-tensor source}

We consider global SH wavefield radiated from a 2-D point seismic source in the model, which corresponds to a line source extending infinitely in $z$ direction. The source is incorporated in the calculation by applying body force $f_{z}$ corresponding to specified focal mechanism in Eq. (1) on discretized grids around center of the source. In 2-D cylindrical coordinates, body force corresponding to a combination of moment tensor components $M_{z r}$ and $M_{z \theta}$ around the center of the source $\left(r_{0}, \theta_{0}\right)$ is written as (Wang et al. 2001).

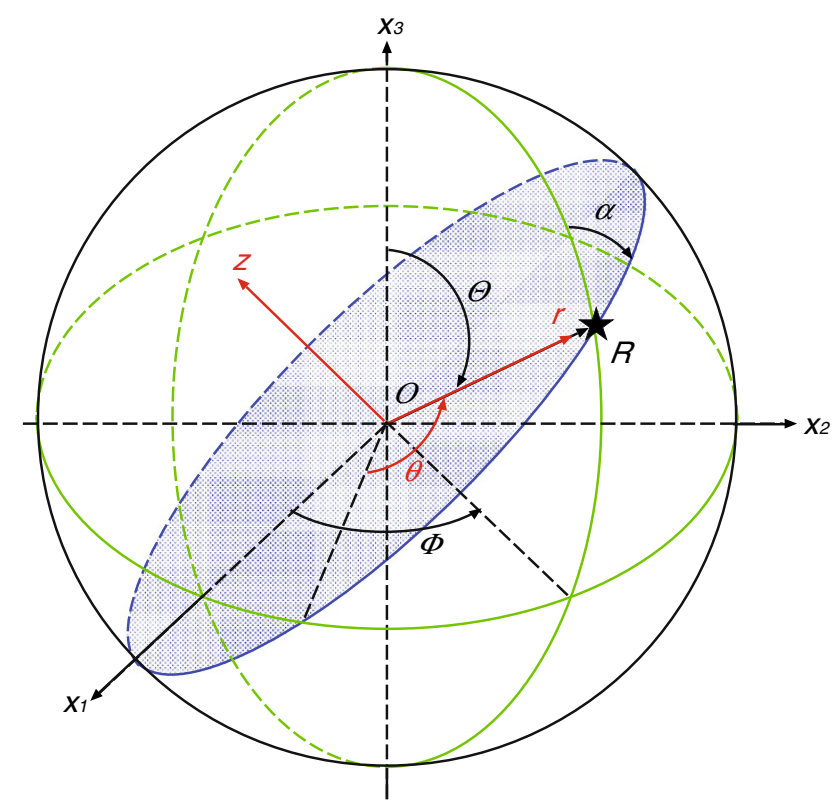

Fig. 2 Transformation from spherical $(R, \Theta, \Phi)$ to cylindrical $(r, \theta$, z) coordinates at source (star). The blue-shadowed plane is our 2-D whole-Earth model, which is a cross section along a great circle of the Earth passing through the source and receiver. $\alpha$ is the azimuth for the source-receiver path at the epicenter

$$
\begin{aligned}
f_{z}(r, \theta, t)= & -M_{z r}(t) \frac{1}{r} \frac{\partial}{\partial r} \delta\left(r-r_{0}\right) \delta\left(\theta-\theta_{0}\right) \\
& -M_{z \theta}(t) \frac{1}{r r_{0}} \frac{\partial}{\partial \theta} \delta\left(r-r_{0}\right) \delta\left(\theta-\theta_{0}\right) .
\end{aligned}
$$

In order to consider moment-tensor source in our calculation, we propose a rotation of seismic moment tensor between 3-D spherical model and 2-D model. Focal mechanism of a point source can be expressed as a seismic moment tensor that describes an earthquake source as a sum of three force couples. In 3-D spherical Earth, one convention is to express moment tensor in a localized Cartesian coordinates $(Z, S, E)$ at the source, where $\mathrm{Z}$ is up, $S$ is south and $E$ is east (Aki and Richards 2002). As shown in Fig. 2, our 2-D whole-Earth model is a cross section of the 3-D spherical Earth along the great-circle plane passing the source and receiver. We denote the coordinates at the source corresponding to the cylindrical system as $(r, \theta, z)$. Then, the seismic moment tensor in this system is given by

$\boldsymbol{M}_{\mathbf{c}}=\left[\begin{array}{lll}M_{\theta \theta} & M_{\theta z} & M_{\theta r} \\ M_{z \theta} & M_{z z} & M_{z r} \\ M_{r \theta} & M_{r z} & M_{r r}\end{array}\right]$.

Let $(R, \Theta, \Phi)$ denote $(Z, S, E)$, the moment tensor in 3-D spherical Earth is written as

$\boldsymbol{M}_{\mathbf{s}}=\left[\begin{array}{ccc}M_{\Theta \Theta} & M_{\Theta \Phi} & M_{\Theta R} \\ M_{\Phi \Theta} & M_{\Phi \Phi} & M_{\Phi R} \\ M_{R \Theta} & M_{R \Phi} & M_{R R}\end{array}\right]$. 
We use moment tensor components of $\boldsymbol{M}_{\mathbf{c}}$ in our 2-D calculation, but $\boldsymbol{M}_{\mathrm{s}}$ is usually given for 3-D spherical Erath. Therefore, we have to convert from $\boldsymbol{M}_{\mathbf{s}}$ to $\boldsymbol{M}_{\mathbf{c}}$. This can be performed by a rotation of moment tensor between the two coordinate systems at the source as described by Tape and Tape (2012). As shown in Fig. 2, the counter-clockwise rotation matrix is written as

$$
\begin{aligned}
\boldsymbol{Q} & =\left[\begin{array}{ccc}
\cos (\pi-\alpha) & \sin (\pi-\alpha) & 0 \\
-\sin (\pi-\alpha) & \cos (\pi-\alpha) & 0 \\
0 & 0 & 1
\end{array}\right] \\
& =\left[\begin{array}{ccc}
-\cos \alpha & \sin \alpha & 0 \\
-\sin \alpha & -\cos \alpha & 0 \\
0 & 0 & 1
\end{array}\right]
\end{aligned}
$$

where $\alpha$ is the azimuth for the source-receiver path at the epicenter. Rotation of moment tensor between spherical and cylindrical coordinates at the source is done by

$M_{\mathrm{c}}=Q M_{\mathrm{s}} Q^{\mathrm{T}}$

Inserting Eqs. (9) and (10) into (11), we get moment tensor components in cylindrical coordinate transformed from those in 3-D spherical coordinates as

$$
\begin{aligned}
& M_{\theta \theta}=M_{\Theta \Theta} \cos ^{2} \alpha-2 M_{\Theta \Phi} \sin \alpha \cos \alpha+M_{\Phi \Phi} \sin ^{2} \alpha, \\
& M_{r \theta}=M_{\theta r}=-M_{R \Theta} \cos \alpha+M_{\Phi R} \sin \alpha, \\
& M_{r r}=M_{R R}, \\
& M_{\theta z}=M_{z \theta}=\left(M_{\Theta \Theta}-M_{\Phi \Phi}\right) \sin \alpha \cos \alpha+M_{\Theta \Phi} \cos 2 \alpha, \\
& M_{r z}=M_{z r}=-M_{\Theta R} \sin \alpha-M_{\Phi R} \cos \alpha, \\
& M_{z z}=M_{\Theta \Theta} \sin ^{2} \alpha+2 M_{\Theta \Phi} \sin \alpha \cos \alpha+M_{\Phi \Phi} \cos ^{2} \alpha
\end{aligned}
$$

These relations can be used to derive the corresponding moment tensor components used in our 2-D calculation from any $\boldsymbol{M}_{\mathrm{s}}$.

\section{Accuracy of the algorithm}

In this section, we perform an accuracy test of our parallel hybrid algorithm. The whole-Earth model is defined between $\theta=0^{\circ}-360^{\circ}$ and $r=3221.0-6371.0 \mathrm{~km}$. We discretize this model with 8,192 and 1,260 grid in $\theta$ and $r$ direction, respectively, which results in a radial grid spacing $\Delta r=2.5 \mathrm{~km}$ and a lateral grid spacing varies from $2.47 \mathrm{~km}$ at the bottom to $4.89 \mathrm{~km}$ at the Earth's surface. The velocity model used in this modeling is the PREM model. Considering the minimum grid spacing and the maximum shear velocity in the model, we take a time interval of $0.025 \mathrm{~s}$ given by the stability condition. The source time function is a bell-shaped function with width of $30 \mathrm{~s}$. We calculate for 120,000 time steps to give synthetic waveforms of $3,000 \mathrm{~s}$ duration. The whole model is

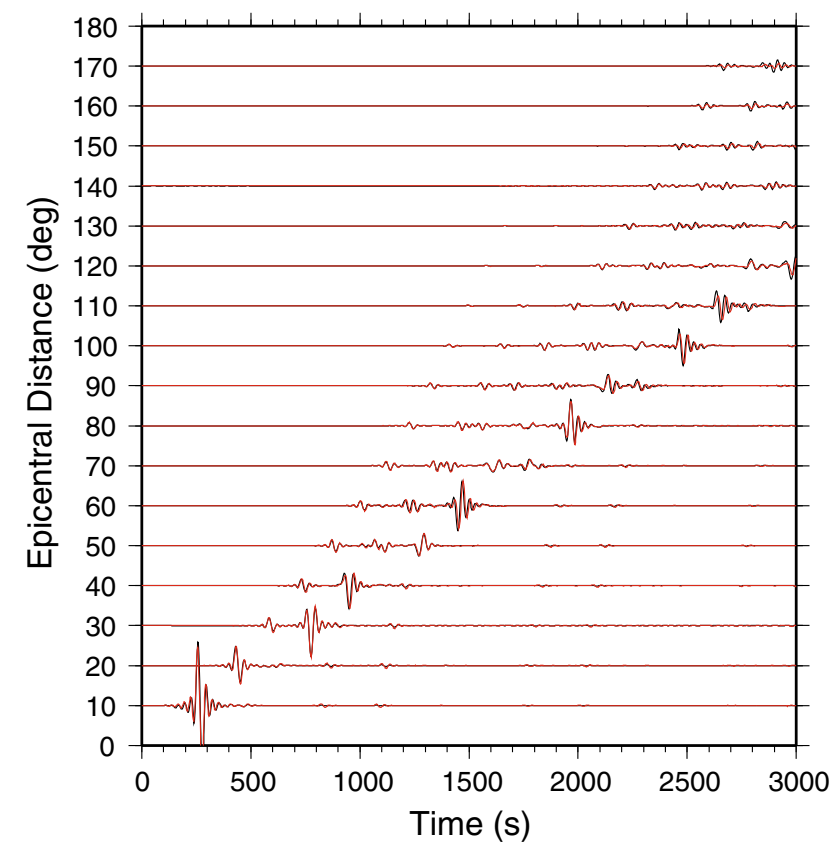

Fig. 3 Comparison of velocity seismograms calculated with our parallel hybrid PSM/FDM algorithm (red lines) and those from DSM (back lines). A low-pass filter $(<1 / 30 \mathrm{~Hz})$ has been applied for all traces

divided into 30 sub-domains and assigned to 30 processors on a PC cluster.

We compare our synthetic waveforms with those obtained by the DSM (Cummins et al. 1997) that gives exact waveforms for spherically symmetric media. The DSM results are calculated for a $600-\mathrm{km}$-deep source with moment tensor component $M_{\Theta \Phi}=1.0 \times 10^{25}$ dyne $\cdot \mathrm{cm}$ (all other components are zeros). The receivers are located at the surface of a great circle with azimuth $\alpha=0^{\circ}$. Applying, moment tensor rotation using equation (12), we get the corresponding moment tensor component for our 2-D model is $M_{\theta z}=1.0 \times 10^{25} \mathrm{dyne} \cdot \mathrm{cm}$.

Figure 3 shows the velocity $\left(v_{z}\right)$ waveforms calculated with our algorithm together with DSM results. For comparison, we compensate the differences between 2-D and 3-D geometric spreading and 2-D line and 3-D point source radiation for our results by applying a correction coefficient used by Wang et al. (2001). We see a very good agreement of waveforms and arrival times for nearly all seismic phases between the two results. At $170^{\circ}$, the waveforms calculated with our method show little amplitude reduction and phase shift comparing with the DSM result. Such a difference for the surface multiple reflections is caused by the numerical dispersion and errors accumulated after propagation for a very long distance that can be reduced with a denser grid spacing than that used in the present modeling. This comparison suggests that our parallel 


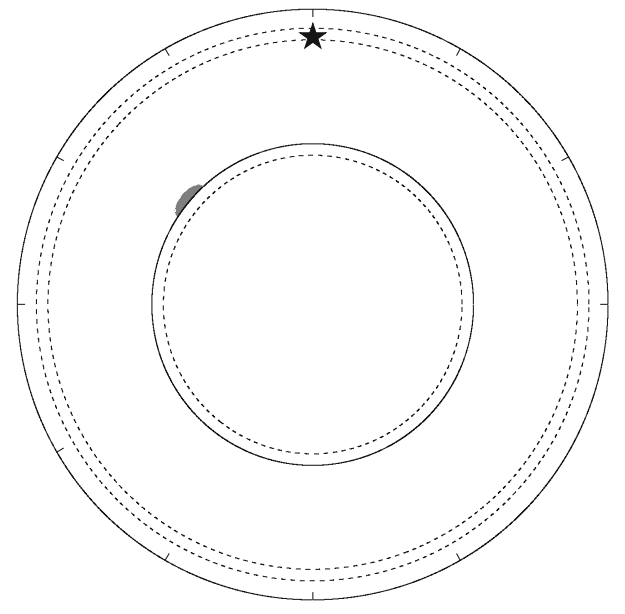

Fig. 4 The lateral heterogeneous whole-Earth model. Star represents the 600-km-deep seismic source, and gray region shows the lowvelocity perturbation (5\% velocity reduction relative to PREM) above the CMB. Center of the perturbation is $50^{\circ}$ from the source. Solid and dashed circles from top to bottom are Earth's surface, the CMB, 410 and $660 \mathrm{~km}$ discontinuities and the bottom of the model

hybrid PSM/FDM scheme is valid and can achieve sufficient accuracy.

\section{Application to a lateral heterogeneous model}

We apply our algorithm to a lateral heterogeneous wholeEarth model to illustrate its feasibility. Waveform modeling studies using $\mathrm{SH}$ wave observations have revealed shear wave velocity perturbations at the base of CMB beneath several regions (e.g., Wen 2002; Thorne et al. 2007). In this model, we put a semi-elliptically shaped lowvelocity perturbation region just above the CMB to the background PREM model as shown in Fig. 4. The maximum thickness of the perturbation is $200 \mathrm{~km}$ and length along CMB is $800 \mathrm{~km}$. Center of the low-velocity region is $50^{\circ}$ from the earthquake source. Shear wave velocity reduction in the perturbation region is set to be $5 \%$ relative to background PREM model. We consider a $600-\mathrm{km}$-deep seismic source with moment tensor component $M_{r z}=1.0 \times 10^{25}$ dyne $\cdot \mathrm{cm}$. The whole-Earth model is discretized with the same grid numbers and all other parameters used in the modeling are the same as used in the previous example. The anelastic attenuation of the medium is incorporated into calculation by adopting attenuation coefficients proposed by Graves (1996), which had been successfully used in P-SV wavefield calculation for 2-D whole-Earth model with PSM (Furumura et al. 1998; Wang et al. 2001).

The synthetic velocity waveforms along the Earth's surface calculated for this model are shown in Fig. 5a. At short epicentral distance, we see very strong direct $\mathrm{S}$ wave, and $\mathrm{CMB}$ reflections such as $\mathrm{ScS}, \mathrm{sScS},(\mathrm{ScS})_{2},(\mathrm{sScS})_{2}$ with decaying amplitude. Weak reflections occurred at the 410 and $660 \mathrm{~km}$ discontinuities can be identified between direct $\mathrm{S}$ and $\mathrm{ScS}$. At intermediate epicentral distance, we see strong $\mathrm{sS}$ and reducing arrival time between $\mathrm{S}, \mathrm{ScS}, \mathrm{sS}$, and sScS. At large distance, diffractions at CMB (Sdiff, sSdiff) are visible and multiple reflections occurred at the surface (SS, sSS, SSS) dominate the later part of the waveforms.

In order to examine the effects of low-velocity perturbation on global SH wave propagation; we performed the same modeling for PREM model without heterogeneity. In Fig. 5b, we show differential waveforms obtained by subtracting results of perturbation model from those of PREM. The waveform differences show variations of amplitude, arrival time, and seismic phase caused by the lateral velocity perturbation. We find that the low-velocity region has significant effects on CMB reflections and diffractions at specific epicentral distance. Waveform anomalies for $\mathrm{ScS}$ and $\mathrm{sScS}$ are observed beyond $65^{\circ}$, and become very strong between $100^{\circ}$ and $110^{\circ}$. Differences of CMB diffractions Sdiff and sSdiff can be seen up to about $160^{\circ}$. Significant variations of $(\mathrm{ScS})_{2}$ and $(\mathrm{sScS})_{2}$ appear between $50^{\circ}$ and $80^{\circ}$. Anomalies of multiple CMB reflections can be seen at later time between $50^{\circ}$ and $120^{\circ}$.

\section{Discussion and conclusions}

We proposed a staggered grid hybrid PSM/FDM algorithm for modeling global SH wave propagation in a 2-D axisymmetric whole-Earth model. Comparing with scheme based on PSM (e.g., Wang and Takenaka 2011), FDM adopted in the radial direction treats the free surface condition more realistically and improve computation efficiency by parallel computing on PC cluster. Comparison with DSM for PREM model using a 30 processor cluster shows the high accuracy of our algorithm.

Transformation of seismic moment tensor between 3-D spherical and 2-D cylindrical system were given by a coordinate rotation. We can derive corresponding moment tensor components in our 2-D model from those in 3-D case. Equation (12) shows transformation for all six components in cylindrical coordinates, in which $M_{\theta \theta}, M_{\mathrm{r} \theta}, M_{\mathrm{rr}}$ are defined for 2-D P-SV wavefield modeling such as used by Furumura et al. (1998) and Wang et al. (2001). Therefore, transformation for these components can be readily applied to 2-D global P-SV wave modeling.

Application to a lateral heterogeneous model shows that we can include arbitrary velocity perturbation in the model and study its effects on global SH wave propagation. This suggests the possibility to apply our algorithm to study 
(a)

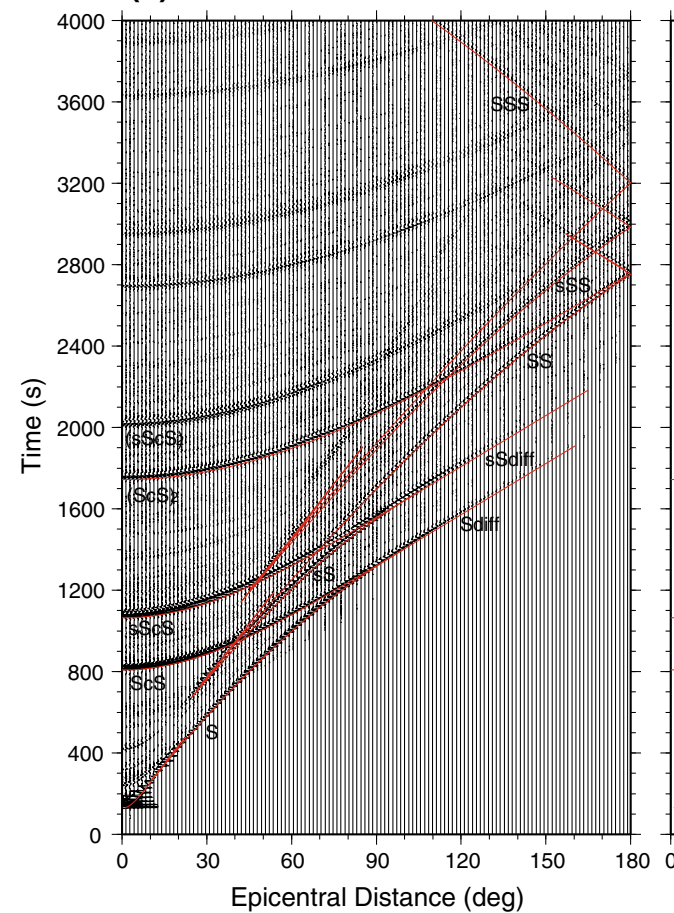

(b)

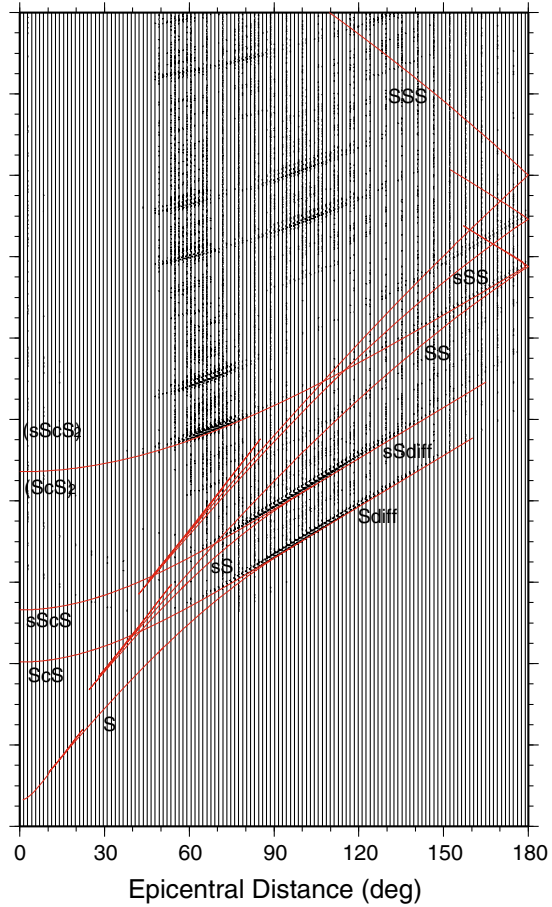

Fig. 5 a Synthetic velocity seismograms at the Earth's surface for the lateral heterogeneous whole-Earth model. b Differential velocity waveforms illustrating anomalies in arrival times and amplitudes caused by the low-velocity perturbation above the CMB. Red curves indicate ray-theoretical arrival times for the marked major phases in the PREM

localized heterogeneity in the Earth by comparing synthetic waveforms and observations.

The algorithm we used for 2-D SH wave modeling in this paper can be extended straightforwardly to 2-D global $\mathrm{P}-\mathrm{SV}$ wave case in a similar 2-D whole-Earth model. With an implementation on PC cluster, 2-D global P-SV wave modeling could be performed with a higher efficiency than previous PSM-based schemes (e.g., Furumura et al. 1998; Wang et al. 2001).

Acknowledgment This research was supported by the National Natural Science Foundation of China (Granted Nos. 41174034 and 40874020).

\section{References}

Aki K, Richards PG (2002) Quantitative seismology, 2nd edn. University Science Books, San Francisco

Cerjan C, Kosloff D, Kosloff R, Reshef M (1985) A nonreflecting boundary condition for discrete acoustic and elastic wave equation. Geophysics 50:705-708

Cummins PR, Takeuchi N, Geller RJ (1997) Computation of complete synthetic seismograms for laterally heterogeneous models using the Direct Solution Method. Geophys J Int 130:1-16

Daudt CR, Braile LW, Nowack RL, Chiang CS (1989) A comparison of finite-difference and Fourier method calculations of synthetic seismograms. Bull Seismol Soc Am 79:1210-1230
Dziewonski AM, Anderson DL (1981) Preliminary reference earth model. Phys Earth Planet Inter 25:297-356

Fornberg B (1996) A practical guide to pseudospectral methods. Cambridge University Press, Cambridge

Furumura T, Kennett BLN, Furumura M (1998) Seismic wavefield calculation for laterally heterogeneous whole earth models using the pseudospectral method. Geophys J Int 135:845-860

Furumura T, Koketsu K, Wen K (2002) Parallel PSM/FDM hybrid simulation of ground motions from the $1999 \mathrm{Chi}-\mathrm{Chi}$, Taiwan, earthquake. Pure Appl Geophys 159:2133-2146

Graves RW (1996) Simulating seismic wave propagation in 3D elastic media using staggered-grid finite differences. Bull Seismol Soc Am 86:1091-1106

Igel H, Gudmundsson O (1997) Frequency-dependent effects on travel times and waveforms of long-period S and SS waves. Phys Earth Planet Inter 104:229-246

Igel H, Weber M (1995) SH-wave propagation in the whole mantle using high-order finite differences. Geophys Res Lett 22:731-734

Igel H, Weber M (1996) P-SV wave propagation in the Earth's mantle using finite differences: application to heterogeneous lowermost mantle structure. Geophys Res Lett 23:415-418

Jahnke G, Thorne MS, Cochard A, Igel H (2008) Global SH-wave propagation using a parallel axisymmetric spherical finitedifference scheme: application to whole mantle scattering. Geophys J Int 173:815-826. doi:10.1111/j.1365-246X.2008. 03744.x

Komatitsch D, Tromp J (2002a) Spectral-element simulations of global seismic wave propagation-I: validation. Geophys J Int. 149:390-412

Komatitsch D, Tromp J (2002b) Spectral-element simulations of global seismic wave propagation-II: three-dimensional models, oceans, rotation and self-gravitation. Geophys J Int 150:303-318 
Nissen-Meyer T, Dahlen FA, Fournier A (2007a) Spherical-Earth Fréchet sensitivity kernels. Geophys J Int 168:1051-1066

Nissen-Meyer T, Fournier A, Dahlen FA (2007b) A 2-D spectralelement method for computing spherical-earth seismograms-I: moment-tensor source. Geophys J Int 168:1067-1093

Özdenva T, McMechan GA (1996) Causes and reduction of numerical artifacts in pseudo-spectral wavefield extrapolation. Geophys J Int 126:819-828

Qin Y, Wang Y, Takenaka H, Zhang X (2012) Seismic ground motion amplification in a 3D sedimentary basin: effect of the vertical velocity gradient. J Geophys Eng 9:761-772. doi:10.1088/17422132/9/6/761

Takeuchi N, Geller RJ, Cummins PR (2000) Complete synthetic seismograms for 3-D heterogeneous Earth models computed using modified DSM operators and their applicability to inversion for Earth structure. Phys Earth Planet Inter 119:25-36

Tape W, Tape C (2012) A geometric setting for moment tensors. Geophys J Int 190:476-498. doi:10.1111/j.1365-246X.2012. 05491.x

Thorne MS, Lay T, Garnero EJ, Jahnke G, Igel H (2007) Seismic imaging of the laterally varying $\mathrm{D}^{\prime \prime}$ region beneath the Cocos Plate. Geophys J Int 170:635-648. doi:10.1111/j.1365-246X. 2006.03279.x

Toyokuni G, Takenaka H (2006) FDM computation of seismic wavefield for an axisymmetric earth with a moment tensor point source. Earth Planets Space 58:e29-e32

Toyokuni G, Takenaka H (2012) Accurate and efficient modeling of global seismic wave propagation for an attenuative Earth model including the center. Phys Earth Planet Inter 200-201:45-55
Toyokuni G, Takenaka H, Wang Y, Kennett BLN (2005) Quasispherical approach for seismic wave modeling in a 2D slice of a global Earth model with lateral heterogeneity. Geophys Res Lett 32:L09305. doi:10.1029/2004GL022180

Virieux J (1986) P-SV wave propagation in heterogeneous media: velocity-stress finite-difference method. Geophysics 51:889-901

Wang Y, Takenaka H (2001) A multidomain approach of the Fourier pseudospectral method using discontinuous grid for elastic wave modeling. Earth Planets Space 53:149-158

Wang Y, Takenaka H (2010) A scheme to treat the singularity in global seismic wavefield simulation using pseudospectral method with staggered grids. Earthq Sci 23:121-127. doi:10. 1007/s11589-010-0001-x

Wang Y, Takenaka H (2011) SH-wavefield simulation for a laterally heterogeneous whole-Earth model using the pseudospectral method. Sci China Earth Sci 54:1940-1947. doi:10.1007/ s11430-011-4244-8

Wang Y, Takenaka H, Furumura T (2001) Modelling seismic wave propagation in a two-dimensional cylindrical whole-earth model using the pseudospectral method. Geophys J Int 145:689-708

Wang Y, Takenaka H, Jiang X, Lei J (2013) Modelling twodimensional global seismic wave propagation in a laterally heterogeneous whole-Moon model. Geophys J Int 192:1271-1287. doi:10.1093/gji/ggs094

Wen L (2002) An SH hybrid method and shear velocity structures in the lowermost mantle beneath the central Pacific and South Atlantic Oceans. J Geophys Res 107:2055. doi:10.1029/2001JB000499

Witte DC, Richards PG (1990) The pseudospectral method for simulating wave propagation. Comput Acoust 3:1-18 